\title{
Impact of Alveolar Recruitment Maneuver in the Postoperative Period of Videolaparoscopic Bariatric Surgery
}

\author{
Paula Patelli Juliani Remístico 1, Sebastião Araújo 2, Luciana Castilho de Figueiredo ${ }^{3}$, Esperidião Elias Aquim 4 , \\ Larissa Mottim Gomes ${ }^{5}$, Morgana Lima Sombrio ${ }^{5}$, Sabrina Donatti Ferreira Ambiel 6
}

Summary: Remístico PPJ, Araújo S, Figueiredo LC, Aquim EE, Gomes LM, Sombrio ML, Ambiel SDF - Impact of Alveolar Recruitment Maneuver in the Postoperative Period of Videolaparoscopic Bariatric Surgery.

Background and objectives: Pulmonary complications in bariatric surgery are common and, therefore, alveolar recruitment maneuvers (ARM) have been used to prevent or reduce them in the postoperative period (POP). The aim of this study was to evaluate the impact of ARM performed intraoperatively in patients undergoing bariatric surgery by videolaparoscopy in the incidence of postoperative pulmonary complications.

Methods: Randomized clinical trial with 30 patients divided into control group (CG) and experimental group (EG), with analysis of spirometric, ventilatory, hemodynamic, and radiographic variables. ARM was performed in EG with positive end expiratory pressure of $30 \mathrm{cmH}_{2} \mathrm{O}$ and inspiratory plateau pressure of $45 \mathrm{cmH}_{2} \mathrm{O}$ for 2 minutes after pneumoperitoneum deflation.

Results: We observed a significant decrease in spirometric values $(p \leq 0.001)$ and higher incidence of pulmonary complications on chest radiograph $(p=0.02)$ in $C G$, as well as significant improvement in dyspnoea Borg scale $(p \leq 0.001)$ in EG.

Conclusions: We conclude that ARM is a safe and effective technique when used for prevention of pulmonary complications in patients undergoing bariatric surgery, resulting in more favorable radiological and spirometric findings in the experimental group compared to the control group in the PO.

Keywords: Positive Pressure Respiration; Postoperative Complications; Pulmonary Atelectasis; Laparoscopy; Bariatric Surgery.

[Rev Bras Anestesiol 2011;61(2): 163-176] @Elsevier Editora Ltda.

\section{INTRODUCTION}

Bariatric surgery by videolaparoscopy has been used since 1994 with a mixed technique of Roux-en-Y gastric bypass as a less invasive procedure, thereby reducing the incidence of early and late complications compared with the conventional technique ${ }^{1-3}$.

Morbid obesity can promote a restrictive syndrome with accumulation of perithoracic and abdominal fat, aggravated after general anesthesia in the supine position, a fact that leads to

\footnotetext{
Received from Hospital Vita Batel in Curitiba, Parana, Brazil.
}

1. Physiotherapist in the Intensive Care Unit of Instituto de Assistência Médica ao Servidor Público Estadual (IAMSPE), M.Sc. Student, Department of Surgery, Faculdade de Ciências Médicas da Universidade Estadual de Campinas (FCM-UNICAMP)

2. Assistant Professor Ph.D. UNICAMP. PhD in Medical Sciences UNICAMP

3. Physiotherapist in the Intensive Care Unit of Hospital das Clínicas, UNICAMP, PhD Department of Surgery, FCM-UNICAMP

4. Physiotherapist, Ph.D., Director of Prófisio Assistência Fisioterápica, Professor of the Lato Sensu Post-graduate Program in Physical Therapy in Intensive Care at the Centro de Estudos, Pesquisa e Extensão em Saúde Inspira, Curitiba, $P R$

5. Physiotherapist; Postgraduate Student at Lato Sensu Post-graduate Program in Physical Therapy in Intensive Care at the Centro de Estudos, Pesquisa e Extensão em Saúde Inspira, Curitiba, $P R$

6. Physiotherapist at Hospital Vita Batel, Specialist in Physical Therapy in Intensive Care at Centro Universitário Campos de Andrade - Uniandrade; Specialist Cardio-Respiratory Physiotherapy at Universidade Tuiuti do Paraná

Submitted on September 11, 2009

Approved on September 9, 2010.

Correspondence to:

Dra. Paula Patelli Juliani Remístico

Faculty of Medical Sciences - Universidade Estadual de Campinas

Rua Tessália Vieira de Camargo, 126

Cidade Universitária "Zeferino Vaz"

13083-887, PO Box: 6111 - Campinas, SP, Brazil

E-mail: paulapatelli@hotmail.com reduced lung volume and functional residual capacity (FRC), favoring the development of atelectasis and changing the relationship between ventilation and perfusion, and increasing the physiological pulmonary shunt ${ }^{4-11}$.

Some ventilatory strategies have been proposed and used to improve gas exchange during anesthesia in bariatric surgery ${ }^{12-14}$. Among them, the most studied today is the recruitment maneuver (ARM) that, which consists of pulmonary inflations and sustained use of positive end expiratory pressure (PEEP) to prevent atelectasis or alveolar hypoventilation during surgery in operated patients ${ }^{15}$.

Therefore, the purpose of this study was to evaluate the impact of recruitment maneuvers performed during surgery on the incidence of postoperative pulmonary complications in patients undergoing videolaparoscopic bariatric surgery.

\section{METHOD}

This was a randomized clinical trial with a cross-analytic and prospective design, with a quantitative approach developed at the Hospital Vita Batel, Curitiba-PR, in the period from February to June 2009. The project was approved by the Research Ethics Committee of Centro de Estudos, Pesquisa e Extensão em Saúde Inspirar (Approval report no 014/08) and a written informed consent term in writing was obtained from each participant.

The criteria used for inclusion in the survey refer to patients undergoing bariatric surgery by videolaparoscopy, of both sexes aged between 20 and 65 . The exclusion criteria 
are patients with serious lung diseases, congestive heart failure (NYHA class III or IV), coronary artery disease, individuals who, for some reason, required intervention by laparotomy, and those who presented hemodynamic instability (MAP $\leq 60$ $\mathrm{mmHg}$ ) at the scheduled time for the ARM.

The final population comprised 30 patients of both sexes submitted to bariatric surgery by mixed videolaparoscopy technique randomly distributed into two simple groups, the Control Group (CG) and Experimental Group (EG).

The evaluation was conducted at three specific times: preoperative (Pre-OP), intraoperative (Intra-OP) and postoperative (Post-OP).

At first (pre-OP), patients underwent a pulmonary function test with Respiradyne - Plus spirometer to check the values of forced expiratory volume in one second $\left(F E V_{1}\right)$, forced vital capacity (FVC), peak expiratory flow (PEF), maximal voluntary ventilation (MVV) and forced expiratory flow $25-75 \%$ $\left(\mathrm{FEF}_{25-75 \%}\right)$, having the best value been used for the three attempts performed ${ }^{16-19}$.

In the second stage (Intra-OP), with patients already in the operating room, we initiated $100 \%$ oxygen by face mask, then proceeding to intravenous induction with sequential administration of midazolam (50-10 mg), hydrochloride of remifentanil (300 mg), propofol (2-3 mg. $\left.\mathrm{kg}^{-1}\right)$ and atracurium (50 mg). Maintenance of anesthesia was performed using propofol from 0.075 to $0.1 \mathrm{mg} \cdot \mathrm{kg}^{-1}$ and remifentanil hydrochloride 0.75 to $1.0 \mu \mathrm{g} \cdot \mathrm{kg}^{-1} \cdot \mathrm{min}^{-1}$.

The EG patients underwent ARM using a PEEP of $30 \mathrm{~cm}$ $\mathrm{H} 2 \mathrm{O}$ and inspiratory plateau pressure of $15 \mathrm{cmH}_{2} \mathrm{O}$ above PEEP for two minutes immediately after abdominal deflation of pneumoperitoneum (PNP) using Takaoka Samurai and Dräger Medical fans, recording ventilatory parameters of tidal volume (TV) used, fraction of inspired oxygen $\left(\mathrm{FiO}_{2}\right)$ and PEEP in the early induction. CG patients did not undergo ARM.

Both groups were monitored and hemodynamic and respiratory function were recorded, such as heart rate (HR), respiratory rate (RR), mean arterial pressure (MAP), oxygen saturation $\left(\mathrm{SpO}_{2}\right)$ and end tidal carbon dioxide partial pressure $\left(\mathrm{P}_{\mathrm{ET}} \mathrm{CO}_{2}\right)$ at four specific times: pre-PNP (five minutes after tracheal intubation), post-PNP (15 minutes after installation of pneumoperitoneum), immediately after ARM and five minutes after ARM. Data were collected using the following equipment: vital sign monitor Datascope Spectrum; Dräger Vamos capnograph, and pulse oximetry by Oxipani Takaoka. Also the time of surgery was documented.

Thereafter, lung function tests were again conducted in the first and second days Post-OP. Chest radiographs were performed in the Pre-OP and Post-OP, and analyzed by researchers in conjunction with the report of a radiologist. The patients underwent daily respiratory and motor physiotherapy, according to the Prófisio protocol; after this procedure, the modified BORG scale for dyspnea (BORGd) was evaluated in both groups.

For data analysis, parametric statistical tests were used as normality was noted using Levene's test, applying the Student's $t$ test and ANOVA. To check the likelihood of development res- piratory complications in both groups, the relative risk was calculated to assess the effectiveness of the treatment employed in this study. The level of significance was $5 \%(p \leq 0.05)$.

\section{RESULTS}

There was homogeneity in the demographic characteristics of the sampling distribution for the 30 patients in the study (15 in each group). The gender distribution of subjects in the control group was composed of 10 females (67\%) and 5 males (33\%) as the experimental group, 11 were females $(73 \%)$ and 4 males (27\%).

Surgical time was significantly higher in CG, with an average of 157 minutes compared to EG, which averaged 109 minutes $(p=0.03)$, as seen in Table I.

Regarding the spirometric variables, we found a decrease in $C G$ from the Pre-OP FVC to the 1st and 2nd Post-OP $(p<0.001)$. As for $\mathrm{FEV}_{1}$, a reduction in the Pre-OP for the 1st and 2nd Post-OP ( $p<0.001)$ was observed. The MVV was reduced from the Pre-OP to the 1 st $(p<0.001)$ and to the 2 nd Post-OP ( $p=0.001)$, as were PEF values for the 1st Post-OP $(p=0.001)$ and the 2nd Post-OP $(p=0.031)$ when compared with the Pre-OP. Finally, there was also a reduction in Pre-OP $\mathrm{FEF}_{25-75 \%}$ for the 1st Post-OP ( $\left.p=0.001\right)$ and the 2nd Post-OP $(p=0.017)$, as shown in Table II and Figure 1.

Analyzing the spirometric values of $E G$, there was a decline in FVC for the 1st Pre-OP ( $p=0.024)$ and for the 2nd Post-OP $(p=0.027)$, as shown in Table II and Figure 1.

In CG, no significant changes in the hemodynamic parameters (HR, MAP, $\mathrm{SpO}_{2}$ and $\mathrm{P}_{\mathrm{ET}} \mathrm{CO}_{2}$ ) were observed, (Table III). In EG, HR was reduced in value by comparing the Pre-PNP and -Post-ARM moments $(p=0.04)$.

The variable $\mathrm{P}_{\mathrm{ET}} \mathrm{CO}_{2}$ showed an increase when comparing the Post-PNP with the Post-ARM moments $(p=0.02)$, as shown in Figure 2 and Table III.

Regarding the Borg dyspnea scale (BORGd), the CG showed a slight but not significant symptom improvement from the 1st to the 2nd Post-OP. On the other hand, the EG showed statistically significant improvement in BORGd from

Table I - Demographic Data of the Studied Population in the Preoperative Period

\begin{tabular}{lccc}
\hline & \multicolumn{2}{c}{ Group } & \\
\cline { 2 - 3 } Variables & Control & Experimental & $\mathrm{p}$ \\
\hline Age(years) & $37.2 \pm 12.2$ & $42.1 \pm 14.5$ & 0.32 \\
BMI & $35.4 \pm 5.5$ & $35.2 \pm 5.5$ & 0.89 \\
Surgery duration & $157 \pm 68.1$ & $109 \pm 47.9$ & $0.03^{*}$ \\
(min) & & & \\
$\mathrm{Vt}$ & $10.8 \pm 1.3$ & $11.5 \pm 2.36$ & 0.30 \\
$\mathrm{FiO}_{2}$ & $0.55 \pm 0.13$ & $0.51 \pm 0.03$ & 0.25 \\
$\mathrm{PEEP}^{\mathrm{RR}}$ & $5.4 \pm 0.91$ & $5.7 \pm 0.9$ & 0.45 \\
& $10.8 \pm 1.3$ & $10.7 \pm 0.9$ & 0.87 \\
\hline
\end{tabular}

Values expressed as mean \pm standard deviation, BMl: body mass index; Vt: tidal volume, $\mathrm{FiO}_{2}$ : fraction of inspired oxygen, PEEP: positive end expiratory pressure; RR: respiratory rate, ${ }^{*} \mathrm{p} \leq 0.05$. 
Table II -Descriptive Data of the Spirometric Test

\begin{tabular}{|c|c|c|c|}
\hline \multirow[b]{2}{*}{ Variables } & \multicolumn{3}{|c|}{ Condition } \\
\hline & Pre & 1st PO & 2nd PO \\
\hline \multicolumn{4}{|l|}{ FVC } \\
\hline Control Group & $3.49 \pm 0.59$ & $2.49 \pm 0.49$ & $2.42 \pm 0.67$ \\
\hline $\begin{array}{l}\text { Experimental } \\
\text { Group }\end{array}$ & $3.49 \pm 0.90$ & $2.61 \pm 1.04$ & $2.65 \pm 1.09$ \\
\hline \multicolumn{4}{|l|}{$\mathrm{FEV}_{1}$} \\
\hline Control Group & $2.95 \pm 0.58$ & $1.98 \pm 0.42$ & $2.04 \pm 0.50$ \\
\hline $\begin{array}{l}\text { Experimental } \\
\text { Group }\end{array}$ & $2.77 \pm 0.98$ & $2.18 \pm 0.98$ & $2.23 \pm 1.01$ \\
\hline \multicolumn{4}{|l|}{ MVV } \\
\hline Control Group & $108.07 \pm 28.65$ & $73.02 \pm 13.73$ & $75.37 \pm 22.19$ \\
\hline $\begin{array}{l}\text { Experimental } \\
\text { Group }\end{array}$ & $106.43 \pm 39.99$ & $83.38 \pm 33.99$ & $83.28 \pm 30.66$ \\
\hline \multicolumn{4}{|l|}{ PEF } \\
\hline Control Group & $350.04 \pm 95.50$ & $228.04 \pm 63.06$ & $267.68 \pm 94.82$ \\
\hline $\begin{array}{l}\text { Experimental } \\
\text { Group }\end{array}$ & $346.42 \pm 174.81$ & $268.77 \pm 155.75$ & $289.47 \pm 166.39$ \\
\hline \multicolumn{4}{|l|}{ FEF $_{25-75 \%}$} \\
\hline Control Group & $3.65 \pm 0.87$ & $2.32 \pm 0.83$ & $2.69 \pm 1.02$ \\
\hline $\begin{array}{l}\text { Experimental } \\
\text { Group }\end{array}$ & $3.32 \pm 1.39$ & $2.49 \pm 1.41$ & $2.97 \pm 1.52$ \\
\hline
\end{tabular}

Values expressed as mean \pm standard deviation; FVC: forced vital capacity, FEV: forced expiratory volume, MVV: maximal voluntary ventilation; PEF: peak expiratory flow, FEF: forced expiratory flow. the 1st to the 2nd Post-OP ( $p<0.001)$, as shown in Table IV and Figure 3.

To assess the risk of patients developing respiratory complications, we calculated the relative risk (RR) and efficacy of treatment. Evaluation of radiographic findings of patients included in the survey did not reveal significant changes in any of the patients in the Pre-AP, whereas in the Post-OP, nine individuals from the control group showed changes $(40 \%$ atelectasis and $20 \%$ pleural effusion), and in EG only two individuals showed changes (13\% pleural effusion).

Calculating the relative risk, with a confidence interval of 95\% (0.06-0.86), it was shown that CG showed an increased risk of developing pulmonary complications in the post-OP (78\% more than the $\mathrm{EG}$; $\mathrm{RR}=0.06-0.86$, confidence interval $95 \%, p=0.02)$.

\section{DISCUSSION}

In the present trial, it was observed that in relation to the ventilatory parameters evaluated ( $\left.\mathrm{VC}, \mathrm{FiO}_{2}, \mathrm{PEEP}, \mathrm{FR}\right)$ no statistically significant differences between groups were found. This might be explained by the standardization of mechanical ventilation used by the staff anesthesiologists. However, the prolonged duration of surgery can cause deleterious effects on lung mechanics ${ }^{20}$, and this may be, partially one explanation for the higher number of respiratory and spirometric changes observed in Post-OP patients in the $\mathrm{CG}$,
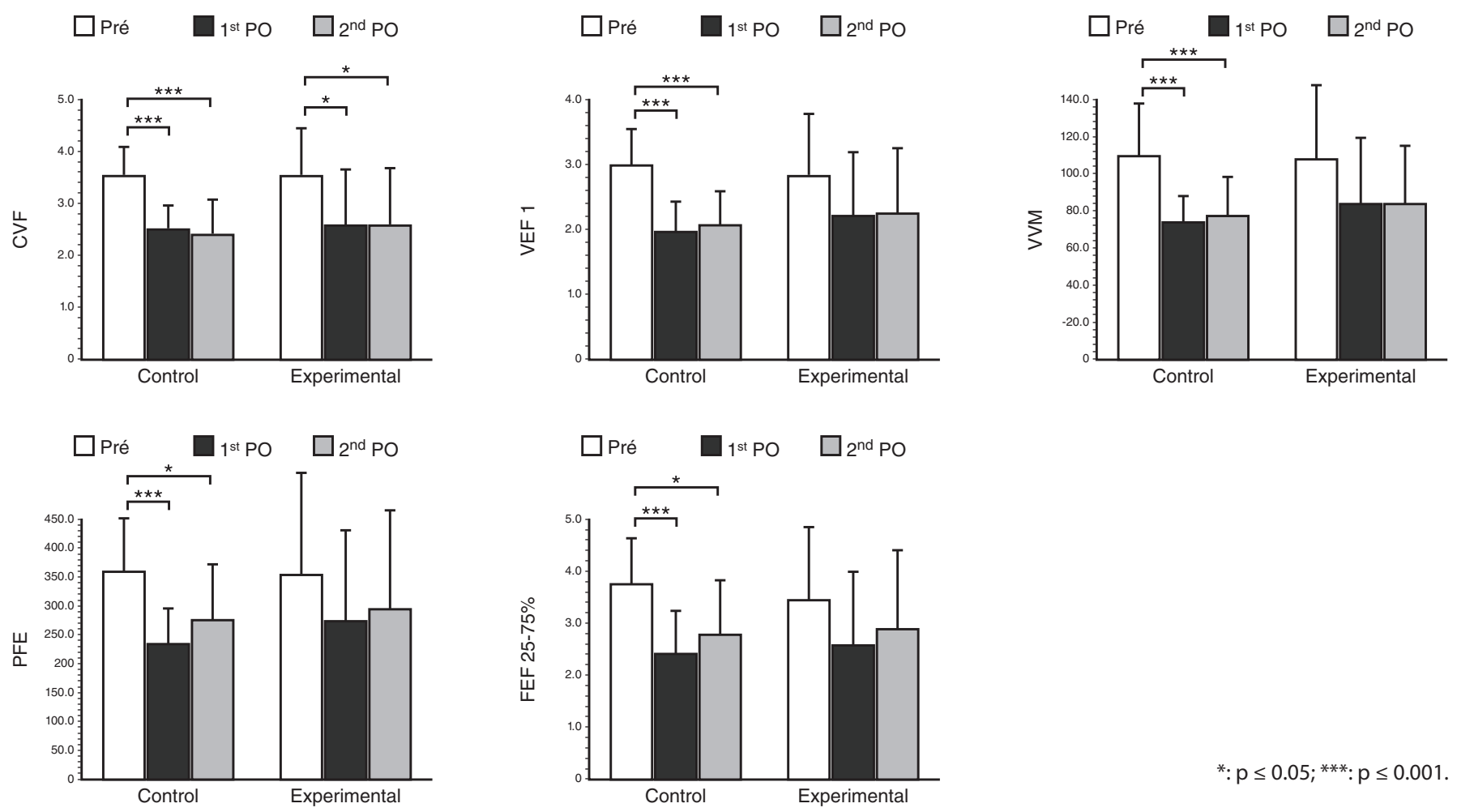

$*: p \leq 0.05 ; * * *: p \leq 0.001$.

Figure 1 - Comparison of variables between groups Spirometry. 
Table III - Descriptive data of the Alveolar Recruitment Maneuver

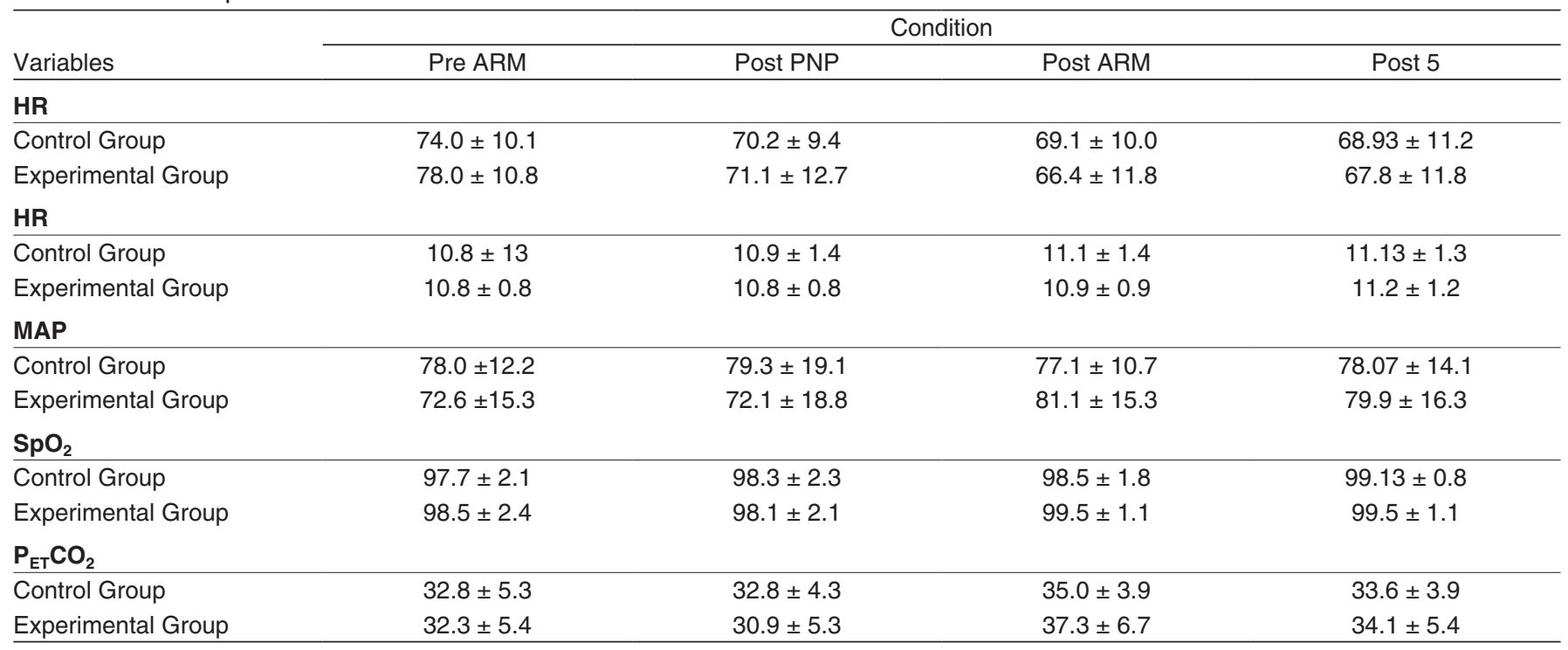

Values expressed as mean \pm standard deviation, $\mathrm{HR}$ : heart rate, RR: respiratory rate, MAP: mean arterial pressure, $\mathrm{SpO}_{2}$, oxygen saturation; $\mathrm{P}_{\mathrm{ET}} \mathrm{CO}_{2}$ : end tidal carbon dioxide.
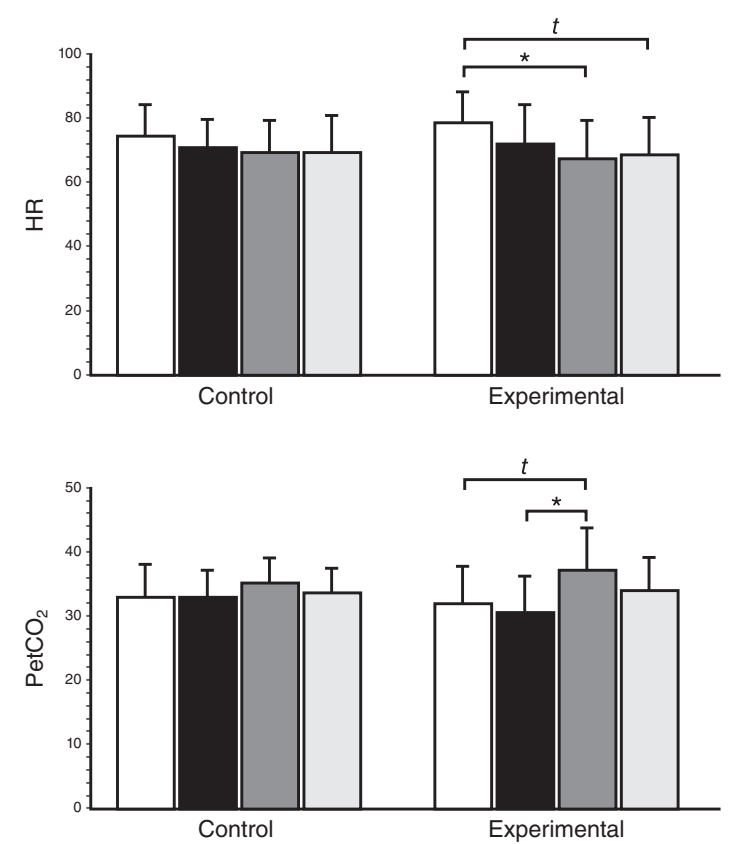

$\square$ Pre ARM $\square$ Post PNP $\square$ Post AR $\square$ Post 5 min

Figure 2 - Comparison of Hemodynamic and Respiratory Variables.

since the surgical time was significantly higher compared to the EG ( $p=0.03)$.

This study demonstrated a reduction in the values planned for the assessment in both groups. In a recent study, Cardoso Filho et al. ${ }^{21}$ demonstrated a decrease in these variables due to the surgery and the anesthetics used, leading to varying degrees of postoperative pain and impaired respiratory muscle
Table IV - Descriptive Data of the Modified Borg Scale Related to Dyspnea

\begin{tabular}{lcc}
\hline & \multicolumn{2}{c}{ Condition } \\
\cline { 2 - 3 } Variables & 1st PO & 2nd PO \\
\hline BORGd & & \\
Control Group & $4.1 \pm 2.25$ & $2.87 \pm 1.92$ \\
Experimental Group & $5.6 \pm 2.41$ & $3.07 \pm 2.12$ \\
\hline
\end{tabular}

Values expressed as mean \pm standard deviation.

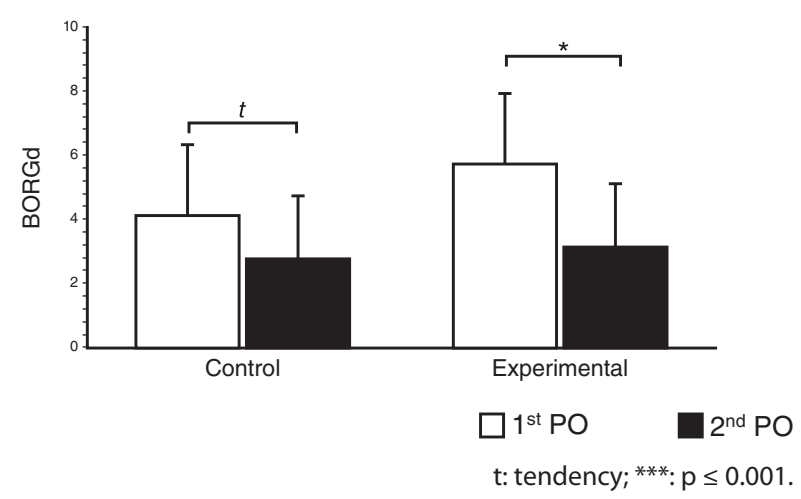

Figure 3 - Analysis According to the BORG Dyspnea Scale.

function. Another group showed a decrease of $51 \%$ of FVC in obese patients as compared to subjects with normal body mass index (BMI) ${ }^{22}$.

The intensity of postoperative pain certainly plays an important role in preservation of pulmonary function after surgery ${ }^{23-26}$, a fact confirmed by the decrease in spirometry values in all patients. 
Eichenberger et al. ${ }^{10}$ demonstrated, by means of computed tomography (CT), areas of atelectasis in morbidly obese patients before induction of anesthesia, after extubation and 24 hours after extubation. The authors concluded that the areas of atelectasis in morbidly obese patients tend to increase and persist 24 hours after extubation compared to obese people and also to patients with normal BMI.

Other findings observed, also by means of CT, that despite the use of oxygen with $100 \%$ concentration, the application of PEEP throughout the induction period almost completely prevents atelectasis formation in morbidly obese patients. The authors also reported that when induction is performed without PEEP, atelectasis may develop within minutes of induction, significantly impairing the blood oxygenation ${ }^{27}$.

The ARM is a technique that uses sustained increase in airway pressures, with the aim of recruiting collapsed alveolar units, increasing lung area available for gas exchange and, therefore, arterial oxygenation ${ }^{15}$. Nowadays, this procedure has been used frequently during surgery 15,28-31.

The literature shows good results when PEEP is used in patients undergoing general anesthesia, whereas areas of atelectasis develop during the anesthetic procedure, leading to impaired gas exchange, increased pulmonary shunt and worsening of oxygenation, facts that are known to be aggravated in morbidly obese patients $10,13,15,26,29,30,32,33$.

A study showed that the alveolar recruitment with moderate levels of PEEP may produce beneficial effects in patients undergoing abdominal, thoracic, or laparoscopic surgery and in patients prone to developing moderate degrees of lung injury after surgical procedures ${ }^{34}$. Other studies have presented findings more favorable to improve oxygenation and blood pressure of oxygen $\left(\mathrm{PaO}_{2}\right)$ in bariatric surgery ${ }^{13,15,27,35,36}$.

Several authors mention the difficulty of making adequate ventilation in morbidly obese patients, due to unawareness of the ideal VC for such individuals ${ }^{13}$, while others search which mode of ventilation is the best one for them. Cadi et al. ${ }^{37}$ compared the effects of pressure controlled ventilation mode (PCV) to the volume controlled ventilation mode (VCV) in airway pressure, blood gases and hemodynamic variables during surgery to laparoscopic surgery. They concluded that the PCV mode improved gas exchange without increasing pressure ventilation or causing any side effects hemodynamics during anesthesia in laparoscopic bariatric surgery.

Thus, some studies are being used with this mode of ventilation in order to assess the respiratory function in the postoperative period of these patients. One proposal is to evaluate the efficacy of recruitment maneuvers in morbid obesity, since this procedure has not been fully disseminated in this population ${ }^{15}$.

Whalen et al. ${ }^{15}$ quantified the effects of maneuver followed by PEEP on oxygenation, lung mechanics and hemodynamics during laparoscopic bariatric surgery. Two groups were studied showing an increase in $\mathrm{PaO}_{2} / \mathrm{FiO}_{2}$ intraoperatively and increased dynamic compliance of respiratory system. But 30 minutes after extubation, there was no significant difference between the control and the recruitment groups. They conclude it would be reasonable to state that more studies are needed to examine whether the use of immediate postextubation strategy of expansion of the lung (CPAP) might improve outcomes in patients undergoing lung in bariatric surgery.

In a recent study, two techniques of ARM were compared in 47 patients using the response $\mathrm{PaO}_{2} / \mathrm{FiO}_{2}$, and the sum $\mathrm{PaO}_{2}$ and $\mathrm{PaCO}_{2}$ in $\mathrm{BMI}$ class III obese in open bariatric surgery. The authors noted that, at the time of ARM, a statistically significant difference was observed with increase in the values of $\mathrm{PaO}_{2}, \mathrm{PaO}_{2} / \mathrm{FiO}_{2}$ ratio, sum of $\mathrm{PaO}_{2}$ and $\mathrm{PaCO}_{2}$, plateau pressure, and mean airway pressure ${ }^{35}$.

Recently, Sprung et al. ${ }^{36}$ observed 17 patients who underwent a recruitment maneuver during bariatric surgery associated with the use of desflurane. Distributed into two groups, those patients who underwent the maneuver showed an increase in dynamic compliance, decreased inspiratory resistance and improvement oxygenation confirmed by a decreased absorption of desflurane, increasing the alveolar concentration of anesthetic. It was concluded that the recruitment maneuver is an effective method to improve oxygenation during surgery. However, the decreased atelectasis indicated by improvement in oxygenation was not sufficient to alter the blood concentrations of desflurane during induction of anesthesia.

The use of CPAP for the reversal of atelectasis, to improve pulmonary exchange and mechanics is well known and widespread in the literature in cases of acute respiratory distress syndrome who resorted to the method of sustained pressure in the airways with levels ranging from 30 to $40 \mathrm{cmH}_{2} \mathrm{O}$ for 30 to 90 seconds $31,38,39$.

Early studies in obese patients demonstrated that CPAP, besides improving oxygenation, restores CRF 40,41 and reduces the risk of acute respiratory distress syndrome after upper abdominal surgery ${ }^{42}$.

A survey of 1,067 patients evaluated the risk of anastomotic dehiscence on those who used CPAP or not. The authors concluded that this method is useful for the treatment of morbidly obese patients in the postoperative gastric bypass Roux-en-Y, which, in addition to improving blood oxygenation in those using the device, showed no relationship between the use of CPAP with anastomotic rupture ${ }^{43}$.

Another study showed that CPAP improved blood oxygenation in obese compared with those who received oxygen therapy with nasal catheter at 4 L. $\mathrm{min}^{-1}$ in moments as before surgery, 30 minutes after admission to the recovery room, post anesthesia , 4 hours and 8 hours after this admission ${ }^{44}$.

In a recent study, Constantin et al. ${ }^{45}$ used a recruiting maneuver with CPAP of $40 \mathrm{cmH}_{2} \mathrm{O}$ for 30 seconds to produce an improvement in $\mathrm{SpO}_{2}$ and $\mathrm{PaO}_{2}$ during periods of 5 and 30 minutes after intubation. Such a maneuver, besides proving to be effective in reducing hypoxemia during anesthesia, was considered an attractive treatment strategy, for it would have previous and noninvasive ventilation, through the support pressure ventilation in the Pre-OP period.

Such citations are similar to findings of the present research, because the $C G$ spirometric values were inferior to $E G$ in the Post-OP, given that the former was not submitted 
to ARM, which enhances the effectiveness of maneuvering among individuals in the EG, which showed improvement in lung volume and capacity, observed in spirometry and chest radiography. When evaluated separately to chest radiographs, we observed a $78 \%$ reduction in the incidence of pulmonary complications in patients of EG in the Post-OP.

Morbid obesity and pneumoperitoneum significantly affect respiratory mechanics, leading to retention of $\mathrm{CO}_{2}$ compared with 14 non-obese subjects, but the CRF is reduced further when the surgery is performed in Trendelenburg associated with videolaparoscopy ${ }^{23}$. Another study analyzed the static compliance (Cst) and respiratory system compliance (sr) in three different positions: supine, Trendelenburg and reverse Trendelenburg. It was observed that Cst,sr supine was lower in morbidly obese patients, not being significant in other positions; when compared to pneumoperitoneum, showed a significant decrease in Cst,sr 14 in all groups. Two other studies showed the same decrease in Cst, with decrease of $40 \%$ after induction of the PNP ${ }^{46,47}$.

The systemic absorption of $\mathrm{CO}_{2}$ in laparoscopic surgery can bring adverse cardiorespiratory and ventilator effects and increase the load by increasing the transperitoneal pressure, opposing the descent of the diaphragm ${ }^{23}$. From this premise, in the present study we observed that immediately after $A R M$, an increase of $\mathrm{P}_{\mathrm{ET}} \mathrm{CO}_{2}$ was observed, justified by the decrease in minute volume during ARM that leads to this momentary retention of $\mathrm{CO}_{2}$, values that returned to normal after five minutes maneuver.

Research conducted with healthy patients showed hemodynamic changes induced by pneumoperitoneum, as for example raised intra-abdominal and intrathoracic pressures, increased heart rate (HR), blood pressure, systemic vascular resistance and pulmonary vascular resistance ${ }^{14,46,48}$. However, in this study, we found that EG had a significant drop in $\mathrm{HR}$, a finding contrary to the authors cited, with no significant hemodynamic instability, keeping normal values of MAP.

By using a PEEP of $30 \mathrm{cmH}_{2} \mathrm{O}$ in obese patients, we can see improvement in $\mathrm{PaO}_{2} / \mathrm{FiO}_{2} 2$ minutes after the suture of the aponeurosis ${ }^{35}$. In another study, which used an inspiratory pressure of $45 \mathrm{cmH}_{2} \mathrm{O}$ in order to analyze the mechanics of ventilation, the $\mathrm{PaO}_{2}, \mathrm{PaCO}_{2}$ and $\mathrm{P}_{\mathrm{ET}} \mathrm{CO}_{2}$, it was observed that, both in the supine position and in the group Tredelenburg, mechanical respiration after induction of the PNP worsened, compared to the baseline ${ }^{49}$.

Thus, we conclude, based on the findings of this study that the ARM is a safe and effective technique when used for prevention of pulmonary complications in patients undergoing laparoscopic bariatric surgery, evidenced by the best spirometric values and radiographic findings postoperatively in $E G$ compared to $\mathrm{CG}$. 